\title{
Form identification in peripheral vision ${ }^{1}$
}

GEORGE W. MENZER AND JOHN B. THURMOND ${ }^{2}$

UNIVERSITY OF LOUISVILLLE

Outlined and solid-surfaced metric histoforms and polygons were employed in an identification task where choice forms were fixed at 0 deg and target forms at six points along the temporal horizontal meridian in the periphery (nasal retina). Accuracy in the identification of solid-surfaced polygons in the far periphery (80 deg from the fovea) was much higher than has been previously reported. Polygons were identified more quickly than histoforms, but a significant difference was not obtained between the speed of identification of outlined and solid shapes. Combined speed and error data indicated that shape (histoform or polygon) is the most salient dimension of a form less than $50 \mathrm{deg}$ from the fovea, whereas beyond 50 deg from the fovea the surface (outlined or solid) of a form is its most salient dimension. Finally, comparisons of the functional relations provided by these performance data with anatomical and physiological data produced the following hypothesis: Spatial summation of rod receptors beyond $20 \mathrm{deg}$ of angular eccentricity in the nasal retina is directly proportional to the density of rod receptors.

The study of form perception in extrafoveal regions of the retina became a topic of interest in visual research in the late 1920s. Geissler (1926) reported that forms presented to peripheral vision were consistently identifiable at $25 \mathrm{deg}$ from the fovea. Zigler et al (1930) observed that forms could not be identified consistently beyond about $30 \mathrm{deg}$ from the fovea. Other investigations (Kleitman \& Blier, 1928; Collier, 1931) attempted to map the peripheral limits of forms. Generally speaking, all of these investigations led to the conclusion that forms could be consistently identified near the fovea, but that a rapid deterioration in identification performance was observed as the form was removed from the fovea in any of the four quadrants of the retina. Universally, the methodology employed in these studies of peripheral form perception was one of absolute identification. That is to say, the Ss in these studies were asked to name the stimulus presented-usually simple geometric shapes with which the $S$ was familiarized prior to the experiments. No adequate control other than the E's monitoring was exercised over the S's eye movements in these studies.

Perception \& Psychophysics, 1970, Vol. 8 (4)
Research on form perception during the last two decades has been largely focused on the quantification of stimuli and on the development of a psychophysics of form (e.g., see Attneave \& Arnoult, 1956; Michels \& Zusne, 1965; Brown \& Owen, 1967). With few exceptions (cf. Handel \& Christ, 1969), the studies conducted on form perception since the early 1930 s have required identifications of stimuli using central viewing. The present study was designed to determine the effects of two variables on the identification of forms presented at selected points in the periphery; namely, the effects of using different kinds of outlined and solid-surfaced shapes. In designing the study, an attempt was made to bring to the study of form perception in the peripheral retina the weight of the recent quantification of stimuli as well as an improved methodology. Accordingly, six-element forms (i.e., histoforms with six columns and polygons with six sides) were employed since perceptual performance seems to be superior with polygons having about six or seven sides relative to the performance obtained with polygons having a lesser or greater number of sides (Crook, 1957; Fisher, 1959; Brown et al, 1962; Michels et al, 1962). Furthermore, metric polygons were used that have been quantified with use of a logic identical to that employed for specifying the uncertainty characteristics of metric histoforms; hence, the two-figure types used were equated in informational content (Thurmond \& Alluisi, 1967).

In view of prior findings, it seemed reasonable to formulate certain hypotheses concerning the effects of the variables used in this study on peripheral form perception. Thus, a progressive deterioration in performance would be expected as a function of removing the form farther and farther from the fovea. Considering the contribution of such factors as jaggedness to the identification of polygons (Brown \& Andrews, 1968), and the consistently demonstrated superior identification performance obtained with polygons relative to histoforms at comparable levels of complexity (Thurmond, 1969; Thurmond \& Hancock, 1969), it seems likely that a similar difference should exist in peripheral vision. However, the prior finding that outlined forms provide superior performance to solid-surfaced forms in foveal vision
(Thurmond \& Hancock, 1969) does not seem tenable with regard to peripheral form perception. On the contrary, the physiology of the peripheral retina would suggest that solid-surfaced forms should optimize the spatial summation effects of the rod receptors that are most numerous in the periphery, and therefore should yield superior performance to outlined forms.

\section{METHOD}

Stimuli

Filled-in (solid) and outlined six-element constrained metric histoforms and polygons were used as stimuli. The metric histoforms are relatively simple shapes; they appeared as solid black bar graphs on a white background and black outlined bar graphs with a white surface that was distinguished from the background by only the contour of the form. Metric polygons are irregular closed shapes similar to those described by Attneave and Arnoult (1956); these forms appeared as solid black hexagonal shapes on a white background and as black outlined hexagonal shapes with a white surface that was also only distinguished from the background by the contour of the form. Both of these types of forms have been used repeatedly in recent years in various studies of form perception (cf. Brown \& Owen, 1967 Evans, 1967; Thurmond \& Hancock, 1969).

The metric histoform stimuli were constructed with the use of a square matrix of six rows and six columns. Each cell in that matrix may assume one of two possible states-"part of the form" (i.e., enclosed by its contour) or "apart from the form" (i.e., not enclosed by its contour). If the column is chosen as the construction unit of the histoform, there are $6^{6}$ possible random histoforms that may be produced. However, histoforms that are redundant relative to these random forms can be generated if the constraining rule is employed that each possible column height appear once, and only once, in each form. The number of forms that may be generated with the use of this constraining sampling rule is 6 ! or 720 . The metric polygon forms were constructed with the use of a circular matrix consisting of six equidistant radii, each of which had been marked off into six points (Thurmond \& Alluisi, 1967). Therefore, the radii and points of the circular matrix are analogs of 
the columns and cells of the square matrix, and the same generalizations about the number and kinds of forms that can be generated from the square matrix also apply to the circular matrix. The population of metric polygons was also selected with a constrained sampling rule. The constraints were the same as those placed on the histoform shapes so that in each polygon no radial extent appeared more than once, and every possible radial extent was represented. One sample of 15 constrained forms was drawn at random from the population of 720 forms to serve as target stimuli. Thus, the target stimuli consisted of 15 different constrained metric histoforms, both solid and outlined, and 15 different constrained polygon analogs, both solid and outlined, or 60 target stimuli in all. From each of the 15 target forms, two constrained metric choice figures were generated.

The choice forms were associated with the targets and arranged in problem form. The target was presented at one of six selected points in the periphery $(5,20,35$, 50,65 , or $80 \mathrm{deg}$ from the fovea) and two other forms, choice forms, were always presented at the fovea $(0 \mathrm{deg})$. The target stimuli were always presented along the horizontal meridian to the temporal field of the retina. In one-third of the problems, the first choice form was identical to the target form, in another one-third the second choice form was identical, and in the remaining one-third of the trials neither of the choice figures was identical to the target form. The $S$ was made aware of the possibility of a "neither" choice by the presence of a blank square of the same dimensions as the underlying histoform cell matrix.

All the stimuli employed in the experiment were accurately hand-drawn from the six-element matrices, spray-painted black or outlined in black marking pen, and fixed to sturdy white background paper. The cell size utilized in the matrix from which the histoform target stimuli were drawn was $12 \mathrm{~mm} \mathrm{sq}$, and that of the choice figures was $2 \mathrm{~mm} \mathrm{sq}$. The point distances on the radii of the circular matrix from which the polygon target stimuli were drawn was $7.2 \mathrm{~mm}$, and that of the choice figures was $1.6 \mathrm{~mm}$. An identical blank square was used as a stimulus for the "neither" choice in conjunction with both the histoform and polygon choice forms. Finally, the visual angle subtended by the histoform target stimuli was approximately $14 \mathrm{deg}$, and approximately $3 \mathrm{deg}$ for the histoform choice stimuli. The visual angle of the polygon target stimuli was approximately $10 \mathrm{deg}$, and approximately $2 \mathrm{deg}$ for the polygon choice stimuli.

\section{Subjects}

A total of 120 students with corrected or uncorrected visual acuity of $20 / 25$ or better from the introductory psychology classes of the University of Louisville ( 80 males, 40 females) served as Ss. They ranged in age from 17 to 29 years, with a median of 19 years.

\section{Apparatus and Procedure}

Ten Ss were assigned randomly to 2 of the 24 experimental conditions. Each S responded to both the 15 histoform and the 15 polygon problems. However, in order to eliminate possible confounding effects of practice, Ss viewed the shapes at one level of peripheral fixation, and they only viewed either solid or outlined forms - a procedure that permitted use of the same shapes in assessing differences between the solid and outlined forms at different peripheral locations. Two practice problems, one with each shape, preceded the 30 experimental problems, and the order of appearance of the problems was counterbalanced over Ss. For one-half the Ss, all the polygons were displayed first and all the histoforms second; for the other half, the order was reversed.

The apparatus to which the stimuli were attached was a Ferree-Rand color perimeter (simplified model) with fixed, constant illumination. The experimental chamber consisted of a $5 \times 7 \mathrm{ft}$ well-ventilated room that was illuminated by the light of a 100-W light bulb passing through the standard blue filter of the color perimeter and by a $7 \frac{1}{2}-\mathrm{W}$ light used to aid the $E$ in presenting the stimuli. The illumination at the surface of the test stimuli (located $33 \mathrm{~cm}$ from the $S$ 's eye) was $3.0 \mathrm{fc}$ as measured by a Weston foot-candle meter (Model 614); thus, illumination of the stimuli was well into the range required for cone functioning (White, 1964).

Prior to the experiment, S's near foveal visual acuity was tested with a Bausch and Lomb Ortho-Rater (Master model). If the S's acuity was $20 / 25$ or better, he was admitted to the experimental chamber and seated in front of the color perimeter. Only the single eye showing the better foveal visual acuity prior to the experiment was used. An eyepatch was placed over the eye having the poorer foveal visual acuity, and the chin rest of the perimeter was adjusted until he was comfortable. A screen was used to obscure the stimuli while they were being secured to the perimeter. A Grass polygraph (Model 5) was used to record electrooculograms in order that $S$ 's eye movements could be monitored. The reference electrode was attached to S's forehead and the active electrode to the temple region adjacent to his eye. He was

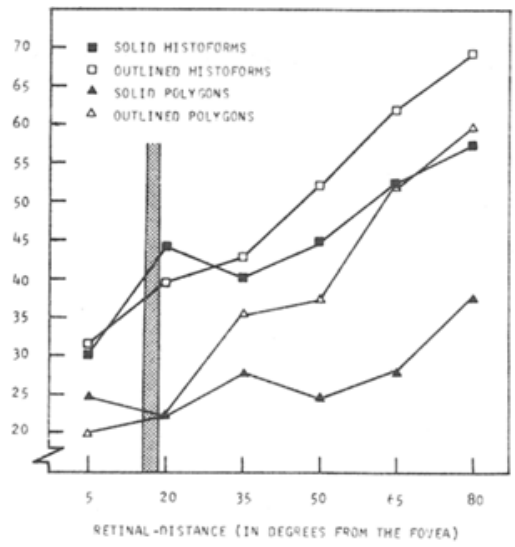

Fig. 1. Mean percentage of errors as a function of retinal distance of solid and outlined forms of both shapes (shaded area represents the location of the blind spot).

instructed always to look straight ahead at the choice figures presented at $\mathbf{0 ~ d e g}$ during the experiment.

Two choice figures and the blank square were presented at 0 deg on a single card (the stimuli were arranged vertically to minimize lateral eye movements), and a target figure was presented at one of the six viewing positions in the periphery. Ss were instructed to respond with the words "one," "two," or "neither," which corresponded to the three choice figures-"one" being the top-most figure, "two" being the middle figure, and "neither" being the bottom-most or blank figure. The instructions emphasized accuracy rather than speed of responding. A voice key consisting of a sound-actuated relay and two amplifiers (Bogen, Challenger model, and Stromberg-Carlson, Model AV29) was used to measure response time. It should be noted that since a forced-choice technique was employed, it was possible for the $S$ to get one-third of the identifications correct by chance alone.

\section{RESULTS}

The mean percetnages of errors are given in Fig. 1 for each of the 24 experimental conditions-six levels of peripheral fixation $(5,20,35,50,65$, or $80 \mathrm{deg})$, two kinds of shapes (histoforms and polygons), and two types of forms (solid and outline). A three-factor analysis of variance computed on the basis of these data indicated that (1) a greater percentage of errors was made in identifying histoforms than in identifying polygons throughout the periphery $(F=103.035, \quad d f=1 / 108$, $\mathrm{p}<.001$ ), (2) fewer errors were made overall with solid than with outlined forms $(F=18.757, \quad d f=1 / 108, p<.001)$, and (3) the percentage of errors was a 
progressively increasing function of the removal of the target figure from the fovea $(F=27.836, \mathrm{df}=5 / 108, \mathrm{p}<.001)$. When the error data of the polygons and histoforms are pooled, the deterioration in performance with both solid and outlined forms is a linear function (see Fig. 3). That is to say, the slopes of the lines of best fit representing these error data were found to be significantly different from zero $(t=16.339, \quad d f=4, p<.01 ; t=5.145$, $\mathrm{df}=4, p<.01$ ) and only a linear fit was statistically significant $\quad(F=174.663$, $\mathrm{df}=1 / 54, \mathrm{p}<.001$ for outlined forms; and $F=17.890, \mathrm{df}=1 / 54, \mathrm{p}<.05$ for solid shapes). It is obvious from examination of Fig. 1, however, that these linear relations are disnupted at the blind spot.

Two of the first-order interactions of the error data were statistically significant, and they may be interpreted with reference to Fig. 1. The difference in overall performance in terms of percentage errors was greater between solid and outlined polygons than between solid and outlined histoforms $(F=4.884, \quad \mathrm{df}=1 / 108$, $\mathrm{p}<.05)$. In addition, the interaction was significant between type of form identified and the dimension of retinal distance $(F=5.285, \quad$ df $=5 / 108, \quad p<.001) . \quad$ The significance of this interaction apparently reflects the fact that generally fewer errors were made with outlined forms near the fovea (at 5 and $20 \mathrm{deg}$ ), whereas beyond $20 \mathrm{deg}$, generally more errors were made with outlined forms.

Inasmuch as accuracy of responding was stressed to the Ss, their error data are probably the more meaningful. However, the mean response times (in seconds) given for each of the 24 experimental conditions in Fig. 2 show functional relations that are essentially identical to those obtained with the error data. Except for the obviously perturbing effects of the blind spot (near $20 \mathrm{deg}$ ), the farther the target stimulus was removed from the fovea, no matter what its type of shape, the longer was the latency of the response to that stimulus $(F=3.142, \quad d f=5 / 108, \quad p<.05)$. The longer response times to histoforms relative to polygons was significant ( $F=100.660$, $\mathrm{df}=1 / 108, \mathrm{p}<.001)$, as was also the interaction between the shape of the figure and its position in the periphery $(F=6.520, \quad \mathrm{df}=5 / 108, \quad p<.001)$. No other effects of the response-time data were significant, but it should be noted that Fig. 2 suggests an especially interesting result; namely, up to $50 \mathrm{deg}$ from the fovea, quicker responses were given to polygons as opposed to histoforms regardless of their surface. Beyond $50 \mathrm{deg}$, quicker responses were obtained with solid forms as opposed to outlined forms for both kinds of shapes.

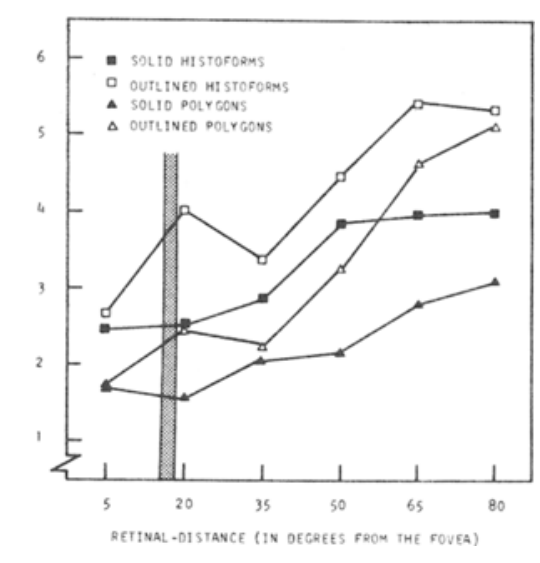

Fig. 2. Mean response time per identification (in seconds) as a function of retinal distance of solid and outlined forms of both shapes (shaded area represents the location of the blind spot).

\section{DISCUSSION}

The data of the present study replicate and extend previous findings concerning form perception in three ways: (1) Performance in the identification of metric polygons is not only superior to the identification of metric histoforms near the fovea (Thurmond, 1969; Thumond \& Hancock, 1969), it is also superior throughout the periphery (2) Identification performance of six-element figures viewed centrally is generally better on outlined than on solid-surfaced shapes (cf. Thurmond \& Hancock, 1969). (3) Performance progressively deteriorates as stimuli are removed from the fovea (Zigler et al, 1930 ; Collier, 1931). In terms of new findings, the results of the present experiment indicate that (4) shapes can be identified much farther into the periphery than previous research had indicated, (5) identification performance with solid-surfaced forms beyond $20 \mathrm{deg}$ from the fovea is considerably better than with outlined forms, and (6) performance on both solid and outlined forms is a decreasing linear function of the degree of removal from the fovea.

The superior identification performance of metric polygons over metric histoforms has been interpreted with respect to differences in the structure of these two kinds of shapes (Thurmond \& Hancock, 1969). Specifically, as Thurmond and Hancock point out, it seems reasonable to assume that man does not process all the information in a figure when perceiving form, but rather that he is more likely to make discriminations on the basis of contour details that most readily distinguish one shape from another (cf. Baker \& Alluisi, 1962). In the case of the histoforms-characterized by a base at the bottom and bars rising-any changes in contour features providing cues for discriminating differences between shapes occur in close spatial proximity. Thus, any particular cue (i.e., any particular element) is embedded in the histoform's contour immediately adjacent to neighboring cues or elements, and the distinctive cue (or cues) for discriminating differences between histoform shapes are masked by adjacent elements. The distinctive contour features of the polygons, on the other hand, are not immediately adjacent to each other; rather, the cues (or elements) for discriminating differences between polygon shapes are spaced around the contour. Therefore, the amount that any particular discriminative cue in the figure's contour is masked by other elements is less for polygons than for histoforms.

The deterioration of performance as stimuli are removed from the fovea noted in earlier research was also found in this study; however, the previous literature suggests that beyond approximately $30 \mathrm{deg}$ from the fovea, little or no form perception is possible (Zigler et al, 1930). Examination of Fig. 1 shows that in the present study, whereas the percentage of errors with histoform and outlined polygon forms was generally quite high in the far periphery, the percentage of errors of identification with solid polygon forms was relatively constant throughout the periphery and reached a high value of only $37.33 \%$ at $80 \mathrm{deg}$ from the fovea. Also, inspection of Fig. 2 shows that the mean response time to solid polygons at $80 \mathrm{deg}$ did not exceed a value of $3 \mathrm{sec}$.

There were, however, a number of important methodological differences between the present study and the prior studies; namely, the previous studies required Ss to make absolute judgments in their identifications of simple outlined geometric forms (e.g., circles, squares, triangles) with which they were usually made familiar before the experimental task was presented. In the present study, both solid and outlined shapes were employed, and Ss were required to make comparative judgments in which the shapes to be identified were presented simultaneously. Furthermore, as was mentioned previously, the six-sided polygons were chosen for use in the present study because in past studies they have consistently produced performance superior to that obtained with polygons having a greater or lesser number of sides. It might also be noted that adequate controls were taken in the present study to insure Ss' foveal fixation, whereas $\mathrm{Ss}^{\prime}$ eye movements were not recorded and monitored in the prior studies. In short, it is apparent that the 


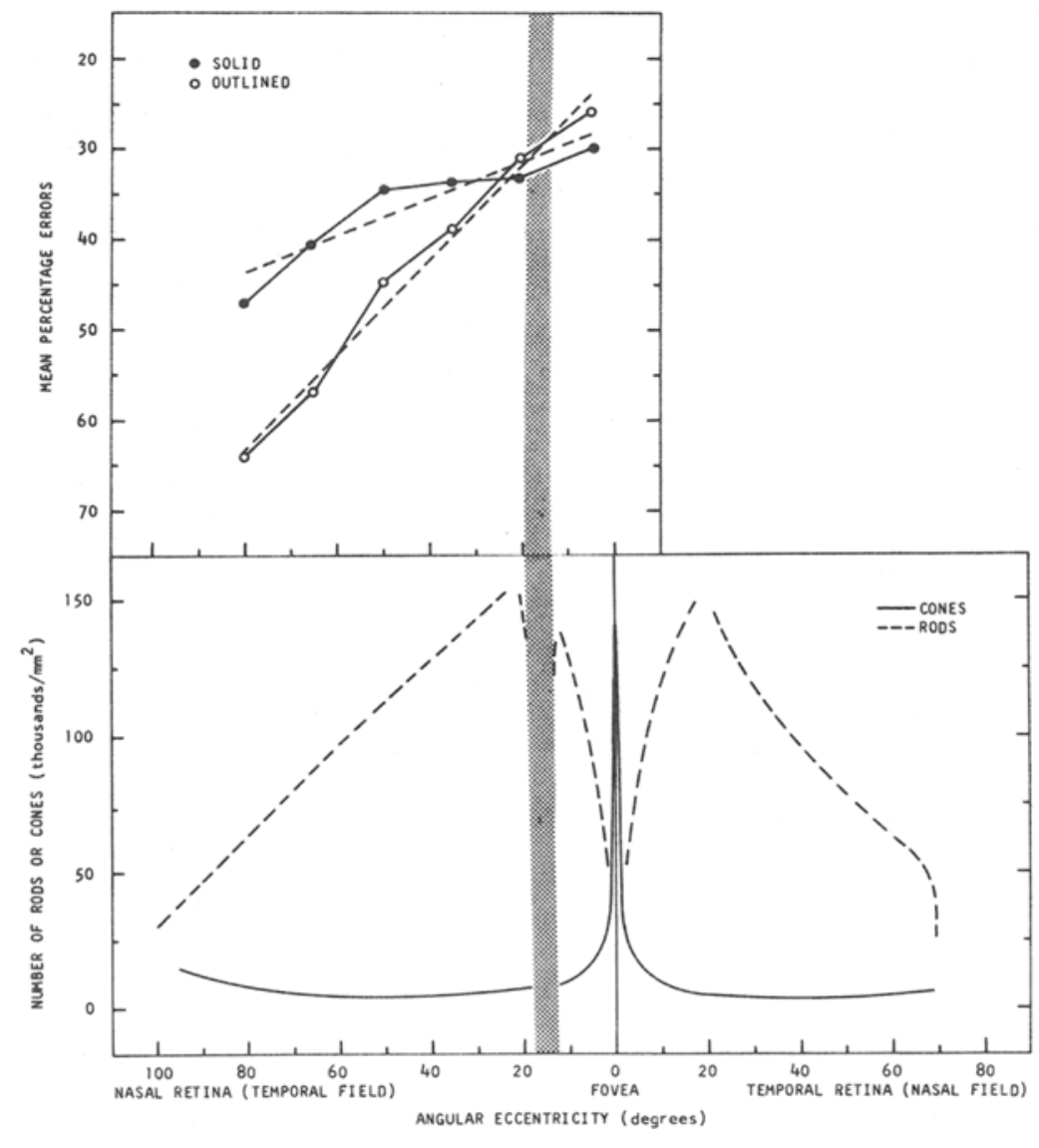

Fig. 3. Mean percentage of errors as a function of retinal distance of solid and outlined forms (upper figure) and the distribution of retinal receptors (lower figure; shaded area represents the location of the blind spot). (Lower figure reprinted with permission. A. Chapanis. How we see. In: National Research Council $A$ survey report on human factors in underseas warfare. Committee on Underseas Warfare, NRC' Washington, D. C. 1949, Chap. 1, (Fig. 2.) ${ }^{3}$

discrepancies between the findings of the present study and those of the prior studies can only be clarified with further experimentation designed to determine certain effects; namely, the effects of using shapes that are less complex than those employed in the present study (e.g., threeor four-element polygons), and the effects of requiring identifications based on absolute as opposed to comparative judgments.

Whereas the shape of a form (histoform or polygon) seems to be its most salient dimension in the range from 0 deg to about $50 \mathrm{deg}$ from the fovea (see Figs. 1 and 2), identification performance was found to be superior on outline shapes when the figures were viewed centrally (i.e., near the fovea at $5 \mathrm{deg}$; see Fig. 3). It is apparent from Figs. 1 and 2 that this result is due to the relatively few errors made on outlined polygons at $5 \mathrm{deg}$ from the fovea. Central foveal viewing (at $0 \mathrm{deg}$ ) in a prior study
(Thurmond \& Hancock, 1969) resulted in superior identification performance on six-element outlined figures in the case of both histoforms and polygons. These data are interpreted generally as supporting the notion that the information relevant to the identification of visual forms is concentrated in the contour of the form (cf. Attneave, 1954). Furthermore, the use of outlined contours apparently enhanced performance by making the contour more distinctive to the relatively dense distribution of cone receptors near the fovea. The cone receptors-characterized by a low degree of spatial summation (cf. Polyak, 1941) and hence a high degree of resolution-may have utilized the outlined contour's "double-edged" cue regarding those points on the contour where contour direction changes most rapidly. Polyak (1941) has suggested that, given the relationship among the receptors in the peripheral retina, it is possible that a stimulus impinging on more than a few rod receptors could sum over those receptors to give a neural response that was greater than would be expected by a stimulus impinging on only a few rods, or a single rod. This assumption, however, has never been tested with as large a stimulus field as was used in the present study. However, it seems reasonable to assume that, beyond $20 \mathrm{deg}$ from the fovea, where the rods are most abundant and cones much less abundant, the solid forms presumably demonstrate superior performance because of the relatively high spatial summation and poor resolution power characteristic of the rod receptors. It is probably the homogeneous dark surface of the solid forms that provided the stimulus for summation in the rod receptors and hence the relatively better performance on the solid forms in peripheral vision. Indeed, the data of Figs. 1 and 2 taken together suggest that beyond $50 \mathrm{deg}$ from the fovea the surface of a form (i.e., whether it is outlined or solid) is its most salient dimension.

The nasal retina (temporal visual field) was employed in this study for the obvious reason that stimuli placed about $30 \mathrm{~cm}$ in front of a human are not visible in his nasal visual field beyond about $30 \mathrm{deg}$ from the fovea. The performance data of the present study shown just above the anatomical data in Fig. 3 demonstrate the linear decrease in accuracy as a function of angular eccentricity for both outlined and solid shapes. Two features concerning the data of Fig. 3 are most striking: (1) The linear lines of best fit for the performance data on outlined and solid shapes cross at approximately $20 \mathrm{deg}$ from the fovea-the degree of angular eccentricity in the anatomical data that corresponds to the bottom of the precipitous drop in cone receptors and the peak in number of rod receptors; (2) As angular eccentricity increases in the nasal retina, a linear function with essentially the same slope describes the decrease in accuracy of identifying outlined shapes and the decrease in the number of rod receptors. This close correspondence in slopes is not unexpected when one considers that the contours of outlined shapes provided little stimulus for spatial summation-i.e., without the confounding effects of spatial summation, the resolution of rod receptors should correlate highly with their density. In support of this interpretation it should be noted that whatever the cone receptors are contributing to these identifications is probably constant, i.e., the cone receptors are relatively low and constant in density between 20 and $80 \mathrm{deg}$ of angular eccentricity. Continuing this line of reasoning, it follows that the linear slope 
describing the decreasing accuracy in identifying solid shapes (see Fig. 3) is less steep owing to the effects of spatial summation in the rod receptors of the nasal retina. Hence on the basis of comparisons between the performance data obtained in the present study and anatomical and physiological data concerning rod and cone receptors, it seems reasonable to formulate the following hypothesis: Spatial summation of rod receptors beyond $20 \mathrm{deg}$ of angular eccentricity in the nasal retina is directly proportional to the density of rod receptors (i.e., spatial summation is a decreasing linear function of angular eccentricity beyond $20 \mathrm{deg}$ in the nasal retina).

These interpetations are admittedly tentative, and it goes without saying that additional research will be necessary to determine the relations between behavioral data, on the one hand, and the anatomy and physiology of the visual system, on the other. One study planned will employ dim illumination (scotopic) conditions in an attempt to isolate the unique contribution of the rod receptors to form identification in peripheral vision. The present study illustrates that the shape and surface of forms presented in peripheral vision are both important determinants of form perception and that these parameters of form seem to be related to the function of the retinal receptors in such a way as to present a complex but understandable basis of form perception in the periphery.

\section{REFERENCES}

ATTNEAVE, $\mathrm{F}$. Some informational aspects of visual perception. Psychological Review, 1954, 61, 183-193.

ATTNEAVE, F, \& ARNOULT, M. D. The quantitative study of shape and pattern perception. Psychological Bulletin, 1956, 53. $452-471$.

BAKER, E. J., \& ALLUISI, E. A. Information handling aspects of visual and auditory form perception. Journal of Engineering Psychology, 1962, 1, 159-179.

BROWN, D. R., \& ANDREWS, M. H. Visual form discrimination: Multidimensional analyses. Perception \& Psychophysics, 1968, 3 401-406.

BROWN, D. R., HITCHCOCK, L., JR., \& MICHELS, K. M. Quantitative studies in form perception: An evaluation of the role of selected stimulus parameters in the visual discrimination performance of human subjects. Perceptual \& Motor Skills, 1962, 14, 519-529.

BROWN, D. R., \& OWEN, D. H. The metrics of visual form: Methodological dyspepsia. Psychological Bulletin, 1967, 68, 243-259.

COLLIER, R. M. An experimental study of form perception in indirect vision. Journal of Comparative Psychology, 1931, 11, 281-289.

CROOK, M. N. Facsimile-generated analogues for instrumental displays. In J. W. Wulfeck and J. H. Taylor (Eds.), Form discrimination as related to military problems. Washington, D.C: National Academy of Sciences-National Research Council, 1957. Pp. 85-98.

EVANS, S. H. VARGUS 7: Computed patterns from Markov processes. Behavioral Science. $1967,12,323-328$.

FISHER, B. The effect of stimulus complexity on form discrimination in Procyon lofor. Unpublished Master's thesis, Purdue University, 1959.

GEISSLER, I. R. Form perception in indirect vision. Psychological Bulletin, 1926, 23, 135-136.

HANDFL, S., \& CHRIST, R. E. Detection and identification of geometric forms using peripheral and central viewing. Perception \& Psychophysics, 1969, 6, 47-49.

KLEITMAN, N., \& BLIER, Z. A. Color and form discrimination in the periphery of the retina American Journal of Physiology, 1928, 85, 178-190.

MICHEIS, K. M., PITTMAN, G. C., HITCHCOCK, L., JR., \& BROWN, D. R. Visual discrimination Tree squirrels and quantified stimulus dimensions. Perceptual \& Motor Skills, $1962,15,443-450$.

MICHFLS, K. M., \& ZUSNE, L. Metrics of visual form. Psychological Bulletin, 1965, 63, 74-86. POLYAK, S. L. The retina. Chicago: The University of Chicago Press, 1941. Pp. 382-383.

THURMOND, J. B. Effects of discrimination difficulty on the identification of different types of visual forms. Psychonomic Science, $1969,15,193-194$.

THURMOND, J. B., \& ALLUISI, E. A. An extension of the information-deductive analysis of form. Psychonomic Science, 1967, 7, 157-158.

THURMOND, J. B., \& HANCOCK, J. B. Effects of figural complexity on the identification of different solid and outlined shapes. Psychonomic Science, 1969, 15, 315-317.

WHITE, W. J. Vision. In P. Webb (Ed.), Bioastronautics data book. Washington, D.C: National Aeronautics and Space Administration, 1964. Pp. 307-341.

ZIGLER, M. J., COOK. B., MILLER, D., \& WEMPLE, L. The perception of form in peripheral vision. American Journal of Psychology, 1930, 42, 246-259.

\section{NOTES}

1. This research was supported in part by the U.S. Army Medical Research and Development Command, Department of the Army, under Contract No. DA-49-193-MD-2567, "Behavioral Effects of Infectious Diseases." This paper is based, in part, on a Master's thesis submitted by the senior author and directed by the junior author at the University of Louisville. An abridged report of this research was presented at the 6lst annual meeting of the Southern Society for Philosophy and Psychology, April 1969 , Miami, Florida. The authors wish to thank Judith A. Menzer for her technical assistance.

2. Address. Performance Research Laboratory, University of Louisville, Louisville, Kentucky 40208.

3. The authors wish to express their appreciation to Professor Alphonse Chapanis and the National Research Council for permission to reprint Fig. 3.

(Accepted for publication December 3, 1969.) 